\title{
INTEGRAL CHARACTERIZATIONS AND THE THEORY OF CURVES
}

\author{
VICTOR DANNON
}

\begin{abstract}
Spherical curves in $E^{4}$ are shown to be given by Frenet-like equations. Thus, finding an integral characterization for a spherical $E^{4}$ curve is identical to finding it for an $E^{3}$ Frenet curve.

For an $E^{3}$ Frenet curve we obtain: Let $\alpha(s)$ be a unit speed $C^{4}$ curve in $E^{3}$ so that $\alpha^{\prime}(s)=T$. Then $\alpha$ is a Frenet curve with curvature $\kappa(s)$ and torsion $\tau(s)$ if and
\end{abstract} only if there are constant vectors $a$ and $b$ so that

$$
\mathbf{T}^{\prime}(s)=\kappa(s)\left\{\mathrm{a} \cos \xi(s)+\mathrm{b} \sin \xi(s)-\int_{0}^{s} \cos [\xi(s)-\xi(\delta)] \mathbf{T}(\delta) \kappa(\delta) d \delta\right\},
$$

where $\xi(s)=\int_{0}^{s} \tau(\delta) d \delta$.

Introduction. The differential equation characterizing a spherical curve is $[1, p$. 62]

$$
\frac{d}{d s}\left\{\frac{1}{\tau(s)} \frac{d \rho}{d s}\right\}+\rho(s) \tau(s)=0,
$$

where $s$ is the length of arc, $\rho(s)=1 / \kappa(s)$ is the radius of curvature and $\tau(s)$ is the torsion of the curve.

In 1971, an explicit solution was first given by Breuer and Gottlieb [4],

$$
\rho(s)=a \cos \int_{0}^{s} \tau(\delta) d \delta+b \sin \int_{0}^{s} \tau(\delta) d \delta,
$$

where $a$ and $b$ are arbitrary constants.

A year later Wong [5] observed that whereas the differential equation holds true only locally (for $\tau \neq 0$ ), the Breuer-Gottlieb solution holds regardless of a possible vanishing of $\tau$ at some point and is therefore a global characterization of a spherical curve.

Wong's observation was derived using a global differential condition of his [3] then an $E^{3}$ curve lies on a sphere if and only if there is a function $f \in C^{1}$ so that $f_{\tau}=(1 / \kappa)^{\prime} ; f^{\prime}+\tau \kappa=0$.

Set $\rho=1 / \kappa$ and consider those equations rewritten in the form

$$
\rho^{\prime}=\tau f, \quad f^{\prime}=-\tau \rho .
$$

We are certainly reminded of the Frenet equations in the plane and this raises the following questions.

(I) Is the Frenet pattern characteristic of a spherical curve in $E^{n}$ ? If so, the problem of getting an integral characterization to a spherical curve is nothing else

Received by the editors October 30, 1979 and, in revised form, July 25, 1980.

1980 Mathematics Subject Classification. Primary 53A04. 
but integration of Frenet equations. (In particular the Breuer-Gottlieb characterization is isomorphic to the integral characterization of Frenet equations in the plane.)

(II) Can the methods (used to obtain characterizations of spherical curves) be extended to include Frenet equations in $E^{3}$ ?

We answer these questions in the affirmative and derive the integral characterizations for an $E^{3}$ Frenet curve.

$S^{3}$ curves and $E^{3}$ Frenet curves. Extension of Wong's condition [3] to $E^{4}$ reveals the connection between $S^{3}$ curves and $E^{3}$ Frenet curves.

In $E^{4}$, denote by $T_{4}$ the fourth orthonormal vector to $T_{1}=T, T_{2}=N, T_{3}=B$ $\left(\mu(s)=\left(B^{\prime}, T_{4}\right)\right.$ measures the change of direction of the space spanned by $\left.T, N, B\right)$. We then have

Proposition 1. Let $\alpha(s)$ be an $E^{4}$ unit speed $C^{5}$ Frenet curve with curvature functions $\kappa(s), \tau(s), \mu(s)$. Then the following are equivalent.

(I) $\alpha(s)$ lies on an $E^{4}$ sphere.

(II) $\kappa(s) \neq 0$ and there are two $C^{2}$ functions $f(s)$ and $g(s)$ so that

$$
\begin{aligned}
& \rho^{\prime}=\tau f, \quad(\rho=1 / \kappa), \\
& f^{\prime}=-\tau \rho+\mu g, \quad g^{\prime}=-\mu f .
\end{aligned}
$$

Proof. Assume that $\alpha(s)$ lies on an $E^{4}$ sphere about $\mathbf{x}_{0}$ with radius a. Fix $\mathbf{x}_{0}$ at $\mathbf{0}$. By repeated differentiation of $a^{2}=(\alpha, \alpha)$ and using Frenet equations (similar to [3]), we obtain $\kappa \neq 0$ and $\mathrm{f}^{\prime}=F \mathbf{f}$ where $f_{i}=\left(-\alpha, \mathrm{T}_{i}\right), i=2,3,4,\left(\rho=f_{2}\right)$. Conversely, given $\mathbf{f}^{\prime}=F \mathbf{f}, d\left\{\alpha-\Sigma_{i=2}^{4} f_{i} \mathbf{T}_{i}\right\} / d s=0$. Therefore, $\alpha-\sum_{i=2}^{4} f_{i} \mathbf{T}_{i}=$ constant $\equiv \mathbf{x}_{0}$. Using the orthogonality, $d\left|\boldsymbol{\alpha}-\mathbf{x}_{\mathbf{0}}\right|^{2} / d s=0$.

Thus $\left|\alpha(s)-\mathbf{x}_{0}\right|^{2}=$ const $=a^{2}$, i.e. $\alpha$ lies on the sphere of radius $a$ about $\mathbf{x}_{0}$ and furthermore $|\mathbf{f}|^{2}=a^{2}$.

We see that $E^{4}$ spherical curve equations have the structure of $E^{3}$ Frenet curves. Finding an integral characterization for a spherical $E^{4}$ curve is identical to finding it for an $E^{3}$ Frenet curve. Extending the methods used to obtain spherical curves characterizations we obtain

An integral characterization of $E^{3}$ Frenet curves. The two bottom $E^{3}$ Frenet equations are of the form

$$
g^{\prime}(s)=-\lambda f+\mu h, \quad h^{\prime}(s)=-\mu g .
$$

Assuming that $\mu$ is nonvanishing (the conclusions are free from this assumption) $g^{\prime} / \mu=-(\lambda f / \mu)+h$. Differentiation, then substitution of $h^{\prime}$ and eventually application of the change of variable $\xi(s)=\int_{0}^{s} \mu(\delta) d \delta$, reduce this equation to

$$
\ddot{g}+g=-(\lambda f / \mu)
$$

(where the variable is $\xi$ ). A particular solution for $g(\xi)$ is

$$
-\int_{0}^{s} \cos [\xi(s)-\xi(\delta)] \lambda(\delta) f(\delta) d \delta .
$$


This leads to

Theorem 2. Let $\alpha(s)$ be a $C^{4}$ curve in $E^{3}$ parametrized by its arc length so that $\alpha^{\prime}(s)=\mathbf{T}$. Then the following are equivalent.

(I) $\alpha(s)$ has a Frenet system $\mathrm{T}, \mathrm{N}, \mathrm{B}$, curvature $\kappa(s)$ and torsion $\tau(s)$ that satisfy the Frenet equations

$$
\mathbf{T}^{\prime}(s)=\kappa \mathbf{N}, \quad \mathbf{N}^{\prime}(s)=-\kappa \mathbf{T}+\tau \mathbf{B}, \quad \mathbf{B}^{\prime}(s)=-\tau \mathbf{N} .
$$

(II) There are constant vectors $\mathbf{a}$ and $\mathbf{b}$ so that

$$
\mathbf{T}^{\prime}(s)=\kappa(s)\left\{\mathbf{a} \cos \xi+\mathbf{b} \sin \xi-\int_{0}^{s} \cos [\xi(s)-\xi(\delta)] \mathbf{T}(\delta) \kappa(\delta) d \delta\right\},
$$

where $\xi(s)=\int_{0}^{s} \tau(\delta) d \delta$.

Proof. Suppose the condition holds. Put

$$
\mathbf{N}(s)=-\int_{0}^{s} \cos [\xi(s)-\xi(\delta)] \mathbf{T}(\delta) \kappa(\delta) d \delta+\mathbf{a} \cos \xi+\mathbf{b} \sin \xi
$$

and

$$
\mathbf{B}(s)=-\int_{0}^{s} \sin [\xi(\delta)-\xi(s)] \mathbf{T}(\delta) \kappa(\delta) d \delta-\mathbf{a} \sin \xi+\mathbf{b} \cos \xi .
$$

Then $\mathbf{N}$ and B satisfy the Frenet equations.

Conversely, if the equations hold, the above $\mathbf{N}$ and $\mathbf{B}$ solve the coupled system $N^{\prime}=-\kappa T+\tau B, B^{\prime}=-\tau \mathbf{N}$. The last equation $\kappa \mathbf{N}=\mathbf{T}^{\prime}$ is our condition.

Condition (II) of the theorem has an analogous condition for $S^{3}$ curves.

The method extends to $E^{n}$ Frenet curves through successive application of transformations of the form $\int_{0}^{s} A(\delta) d \delta$ (one would then use the terminology of [2].)

\section{REFERENCES}

1. E. Kreyzig, Differential geometry, Univ. of Toronto Press, Toronto, 1959.

2. Wilhelm Klingenberg, A course in differential geometry, Springer-Verlag, Berlin and New York, 1978.

3. Yung Chow Wong, A global formulation of the condition for a curve to lie in a sphere, Monatsh. Math. 67 (1963), 363-365.

4. S. Breuer and D. Gottlieb, Explicit characterizations of spherical curves, Proc. Amer. Math. Soc. 27 (1971), 126-127.

5. Yung Chow Wong, On an explicit characterization of spherical curves, Proc. Amer. Math. Soc. 34 (1972), 239-242.

Department of Mathematics, University of SOUTh Florma, Tampa, Florma 33620

Current address: 5008 Springwood Drive, Tampa, Florida 33624 